\title{
ON THE METHODS OF RAYLEIGH-RITZ-WEINSTEIN ${ }^{1}$
}

\author{
C. ARF
}

The Rayleigh-Ritz-Weinstein methods are concerned with the connection between eigenvalues of a completely continuous symmetric operator in a Hilbert space and the eigenvalues of its projection into a subspace $\chi$ of $H$ such that the difference $H \ominus \chi=\bar{\chi}$ is finitedimensional.

In this paper we extend some of the known results of Weinstein and Aronszajn to more general symmetric operators and to the case where $\bar{\chi}$ is not necessarily finite-dimensional.

We consider a continuous symmetric operator $Q$ and the solutions of the equation

$$
(Q-\zeta I) x=0
$$

for a given number $\zeta$. Because of the continuity of $Q$, those solutions form a closed subspace $H_{\zeta}$ of $H$. If $H_{\zeta}$ is not empty, the number $\zeta$ is called an eigenvalue of $Q$ and the elements of $H_{\zeta}$ the eigenvectors of $Q$ corresponding to this eigenvalue. Because of the symmetry of $Q$ the subspace $H_{\zeta}$ is empty for every nonreal $\zeta$. Let us put $\bar{H}_{\zeta}=H \ominus H_{\zeta}$. We designate by $P_{\zeta}$ and $\bar{P}_{\zeta}$ the projectors associated with the subspaces $H_{\zeta}$ and $\bar{H}_{\zeta}$ respectively. Because of the symmetry of $Q$, the operator $Q-I \zeta$ transforms the whole space $H$ into a subspace of $\bar{H}_{\zeta}$. We shall assume that this subspace is identical with $\bar{H}_{5}$, such that there is a self-adjoint operator $R_{\zeta}^{\prime}$ which transforms $H$ into $\bar{H}_{\zeta}$ and which satisfies the conditions

$$
(Q-I \zeta) R_{\zeta}^{\prime}=R_{\zeta}^{\prime}(Q-I \zeta)=\bar{P}_{\zeta} .
$$

We assume further that $R_{\xi}^{\prime}$ is continuous. Let $r$ be a positive number such that

$$
\left\|R_{\zeta}^{\prime} x\right\|<r\|x\|
$$

holds for every $x$ in $H$. The series.

$$
\bar{P}_{\zeta}+(z-\zeta) R_{\zeta}^{\prime}+(z-\zeta)^{2} R_{\zeta}^{\prime 2}+\cdots+(z-\zeta)^{n} R_{\zeta}^{\prime n}+\cdots
$$

then converges uniformly for $|z-\zeta| \leqq r$ and represents a continuous operator which satisfies the equation

$$
\left(\bar{P}_{\zeta}-(z-\zeta) R_{\zeta}^{\prime}\right)\left(\bar{P}_{\zeta}+(z-\zeta) R_{\zeta}^{\prime}+\cdots+(z-\zeta)^{n} R_{\zeta}^{\prime n}+\cdots\right)=\bar{P}_{\zeta} .
$$

Presented to the Society, April 27, 1951; received by the editors June 13, 1951.

1 This work was sponsored by the Office of Naval Research. 
The operator $(Q-I z)=(Q-I \zeta)-(z-\zeta) I$ then has an inverse $R_{z}$ which is represented by

$$
R_{z}=-\frac{P_{\zeta}}{z-\zeta}+\sum_{i=0}^{\infty} R_{\zeta}^{\prime i+1}(z-\zeta)^{i}
$$

for every $z$ which satisfies the inequality $0<|z-\zeta| \leqq r$.

As an example of operators which satisfy the conditions stated above, without necessarily being completely continuous, we can consider the operator

$$
Q=\sum_{i=1}^{\infty} k_{i} P_{i}
$$

where $\left(k_{1}, k_{2}, \cdots, k_{n}, \cdots\right)$ is a bounded set of real numbers and $P_{1}, \quad P_{2}, \cdots, P_{n}, \cdots$ a complete set of orthogonal projectors $\left(\sum_{i=1}^{\infty} P_{i}=I, P_{i} P_{j}=P_{j} P_{i}=0\right.$ for $\left.i \neq j\right)$. This operator obviously satisfies our conditions for every $\zeta$ which is not a limit point of the set $\left(k_{1}, k_{2}, \cdots, k_{n}, \cdots\right)$. In particular we have for this operator $Q, R_{\zeta}^{\prime}=\sum_{k_{i} \neq \zeta} P_{i} /\left(k_{i}-\zeta\right)$.

1. Weinstein's problem. Let $\bar{\chi}$ be a given closed subspace of $H$, $\chi=H \Theta \bar{\chi}$. Find the solutions of the equation

$$
P(Q-I \zeta) P x=0
$$

where $P$ is the projector associated to the subspace $\chi=P H$.

Since $P(Q-I \zeta) P$ is continuous, the set of all solutions of (2) is a closed subspace $\chi_{\zeta}$ of $\chi$.

We designate by $\bar{P}$ the projector $\bar{P}=I-P$ associated to the subspace $\bar{\chi}=\bar{P} H$ and consider the identity

$$
\begin{aligned}
(Q-I \zeta) x \equiv & P(Q-I \zeta) P x+\bar{P}(Q-I \zeta) P x \\
& +P(Q-I \zeta) \bar{P} x+\bar{P}(Q-I \zeta) \bar{P} x
\end{aligned}
$$

which connects the operator $Q-I \zeta$ with the operator $P(Q-I \zeta) P$. Hence $x$ is an element of $\chi_{5}$ if and only if

$$
\begin{aligned}
(Q-I \zeta) x & =\bar{P}(Q-I \zeta) x \\
\bar{P} x & =0
\end{aligned}
$$

According to the equation (4), $x^{\prime}=(Q-I \zeta) x$ is an element of

$$
\bar{\chi}^{\prime}=\bar{H}_{\zeta} \cap \bar{\chi} \text {. }
$$

Let us put

$$
\bar{x}^{\prime \prime}=\bar{x} \ominus \bar{x}^{\prime}
$$


We designate by $\bar{P}^{\prime}$ and $\bar{P}^{\prime \prime}$ the projectors which correspond respectively to the subspaces $\bar{\chi}^{\prime}$ and $\bar{\chi}^{\prime \prime} . x^{\prime}$ is then of the form $x^{\prime}=\bar{P}^{\prime} y$ where $y$ is defined up to addition of an element of $\bar{\chi}^{\prime \prime} \oplus \chi$. Since $x$ is an element of $\chi$, we can choose this $y$ in such a way that

$$
P_{\zeta} x=P_{\zeta} y \text {. }
$$

The elements $x$ of $\chi_{5}$ are therefore of the form

$$
x=R_{\zeta}^{\prime} \bar{P}^{\prime} y+P_{\zeta} y .
$$

Conversely it is easy to verify that every element of the form (6) satisfies the equation (4). The subspace $\chi_{5}$ is therefore the set of all those elements of the form (6), which satisfy the equation

$$
\bar{P} R_{\zeta}^{\prime} \bar{P}^{\prime} y+\bar{P} P_{\zeta} y=0 \text {. }
$$

Let us consider first the elements of $\chi_{\zeta}$ which are of the form $P_{\zeta} y$. It is clear that those elements are in

$$
\chi \cap H_{\zeta}=H_{\zeta}^{\prime}=\chi^{\prime}
$$

and that every element of this subspace $\chi^{\prime}$ is an element of the form $P_{\zeta} y$ of $\chi_{\zeta}$.

REMARK. Since $H_{\zeta}^{\prime}=\chi^{\prime}$ is the subset of all elements of $H_{\zeta}$ orthogonal to $\bar{\chi}$, it is also the subset of all elements of $H_{\zeta}$ orthogonal to

$$
P_{\zeta} \bar{\chi} \subseteq H_{\zeta}
$$

We have therefore

$$
P_{\zeta} \bar{\chi}=H_{\zeta} \ominus H_{\zeta}^{\prime}=H_{\zeta}^{\prime \prime} .
$$

On the other hand, since $\bar{\chi}^{\prime}$ is the set of all elements of $\bar{\chi}$ orthogonal to $H_{\zeta}$, we have

$$
H_{\zeta}^{\prime \prime}=P_{\zeta} \bar{\chi}=P_{\zeta} \bar{\chi}^{\prime \prime}
$$

and

$$
\bar{\chi}^{\prime \prime}=\bar{P} H_{\zeta}=\bar{P} H_{\zeta}^{\prime \prime},
$$

and the correspondences

$$
\begin{array}{ll}
x \leftrightarrow y=P_{\zeta} x, & x \in \bar{x}^{\prime \prime}, y \in H_{\zeta}^{\prime \prime}, \\
y \leftrightarrow x=\bar{P} y, & x \in \bar{\chi}^{\prime \prime}, y \in H_{\zeta}^{\prime \prime},
\end{array}
$$

are one-to-one correspondences. The subspace $H_{\zeta}$ is therefore the direct sum of two subspaces, of which one is the subspace $H_{\zeta}^{\prime}=\chi^{\prime}=\chi \cap H_{\zeta}$ and the other $H_{\zeta}^{\prime \prime}$ which is isomorphic to $\bar{\chi}^{\prime \prime}=\bar{\chi} \theta\left(\bar{H}_{\zeta} \cap \bar{\chi}\right)$. 
Since

$$
H=\bar{\chi} \oplus \chi=\bar{\chi}^{\prime} \oplus \bar{\chi}^{\prime \prime} \oplus \chi^{\prime} \oplus \chi^{\prime \prime} \text { with } \chi^{\prime \prime}=\chi \ominus \chi^{\prime}
$$

we can replace, in the expression (6) of the element $x$ of $\chi_{5}$, the element $y$ by the sum $y=y_{1}+y_{2}+y_{3}+y_{4}$ of four orthogonal vectors $y_{1} \in \bar{\chi}^{\prime}$, $y_{2} \in \bar{\chi}^{\prime \prime}, y_{3} \in \chi^{\prime \prime}, y_{4} \in \chi^{\prime}$. In this way we obtain

$$
x=R_{\zeta}^{\prime} y_{1}+P_{\zeta}\left(y_{2}+y_{3}\right)+y_{4} .
$$

The three terms of this expression are obviously orthogonal to each other. We have therefore

$$
\chi_{\zeta}=\left\{\left[R_{\zeta}^{\prime} \bar{\chi}^{\prime} \oplus P_{\zeta}\left(\bar{\chi}^{\prime \prime} \oplus \chi^{\prime \prime}\right)\right] \cap \chi\right\} \oplus \chi^{\prime} .
$$

Since obviously

$$
H_{\zeta}^{\prime \prime}=P_{\zeta} \bar{\chi}^{\prime \prime}=P_{\zeta}\left(\bar{\chi}^{\prime \prime} \oplus \chi^{\prime \prime}\right) \quad\left(P_{\zeta} \chi^{\prime \prime} \subset H_{\zeta}^{\prime \prime}=H_{\zeta} \ominus \chi^{\prime}\right),
$$

we can write the preceding relation as follows

$$
\chi_{\zeta}=\left\{\left[R_{\zeta}^{\prime} \bar{\chi}^{\prime} \oplus P_{\zeta} \bar{\chi}^{\prime \prime}\right] \cap \chi\right\} \oplus \chi^{\prime} .
$$

The subspace $\chi_{\xi} \ominus \chi^{\prime}$ is therefore the set of all elements of the form

$$
R_{\zeta}^{\prime} y+y^{\prime}, \quad y \in \bar{\chi}^{\prime}, y^{\prime} \in P_{\zeta} \bar{\chi}^{\prime \prime}=H_{\zeta}^{\prime \prime},
$$

which satisfy the condition

$$
\bar{P} R_{\zeta}^{\prime} y+\bar{P} y^{\prime}=0 .
$$

Since $\bar{P}=\bar{P}^{\prime}+\bar{P}^{\prime \prime}$ and since $\bar{\chi}^{\prime}$ is orthogonal to $H_{\zeta}$, this equation can be written as a system of two equations

$$
\begin{aligned}
\bar{P}^{\prime} R_{\zeta}^{\prime} \bar{P}^{\prime} y & =0, & y \in \bar{x}^{\prime}, \\
\bar{P} y^{\prime}+\bar{P}^{\prime \prime} R_{\zeta}^{\prime} \bar{P}^{\prime} y & =0, & y^{\prime} \in H_{\zeta}^{\prime \prime} .
\end{aligned}
$$

We noticed before that the correspondence

$$
y^{\prime} \leftrightarrow \bar{P} y^{\prime}
$$

between $y^{\prime} \in H_{\zeta}^{\prime \prime}$ and $\bar{P} y^{\prime} \in \bar{\chi}^{\prime \prime}=\bar{P} H_{\zeta}^{\prime \prime}$ is one-to-one. We can therefore define a continuous linear operator $T$ such that

$$
T \bar{P} y^{\prime}=y^{\prime}, \quad \text { for } y^{\prime} \in H_{\zeta}^{\prime \prime}\left(T \bar{\chi}^{\prime \prime}=H_{\zeta}^{\prime \prime} \subset H_{\zeta}\right) .
$$

So we obtain all solutions of (7) by putting

$$
R_{\zeta}^{\prime} y+y^{\prime}=R_{\zeta}^{\prime} y-T \bar{P}^{\prime \prime} R_{\zeta}^{\prime} y
$$

where $y$ is a solution of (8). Let us designate by $\bar{\chi}_{1}^{\prime}$ the subspace of all elements $y$ of $\bar{\chi}^{\prime}$ which satisfy (8). Then we can write 


$$
\chi_{\zeta} \theta \chi^{\prime}=\left(R_{\xi}^{\prime}-T \bar{P}^{\prime \prime} R_{\xi}^{\prime}\right) \bar{\chi}_{1}^{\prime} .
$$

We remark further that the correspondence

$$
y \leftrightarrow\left(R_{\zeta}^{\prime}-T \bar{P}^{\prime \prime} R_{\zeta}^{\prime}\right) y
$$

between elements of $\bar{\chi}_{1}^{\prime}$ and $\chi_{5} \theta \bar{\chi}^{\prime}$ is one-to-one, since from

$$
\left(R_{\zeta}^{\prime}-T \bar{P}^{\prime \prime} R_{\zeta}^{\prime}\right) y=0
$$

follows

$$
y=(Q-I \zeta)\left(R_{\zeta}^{\prime}-T \bar{P}^{\prime \prime} R_{\zeta}^{\prime}\right) y=0 .
$$

Now we are going to express the result of our analysis in terms of $R_{z}$, which is an analytic function of $z$ for $0<|z-\zeta| \leqq r$. From the representation

$$
R_{z}=-\frac{P_{\zeta}}{z-\zeta}+\sum_{i=0}^{\infty} R_{\zeta}^{i+1}(z-\zeta)^{i}
$$

of this operator, one sees that the space $\bar{\chi}(z)$ of the functions

$$
y(z)=\bar{P} R_{z} \bar{P} y \quad \text { with } y \in \bar{\chi}=\bar{\chi}^{\prime \prime} \oplus \bar{\chi}_{1}^{\prime} \oplus \bar{\chi}_{0}^{\prime}
$$

is the direct sum of three subspaces which are respectively isomorphic to $\bar{\chi}^{\prime \prime}, \bar{\chi}_{0}^{\prime}=\bar{\chi}^{\prime} \theta \bar{\chi}_{1}^{\prime}, \bar{\chi}_{1}^{\prime}$ :

I. The set of all functions of the form

$$
\bar{P} R_{z} \bar{P} y=-\frac{\bar{P} P_{\zeta} \bar{P} y}{z-\zeta}+\sum_{i=0}^{\infty} \bar{P} R_{\zeta}^{\prime i+1} \bar{P}(z-\zeta)^{i} y, \quad y \in \bar{\chi}^{\prime \prime},
$$

which are completely characterized by their principal parts, since the operator $\bar{P} P_{\zeta} \bar{P}$ transforms $\bar{\chi}^{\prime \prime}$ into itself in a one-to-one manner.

II. The set of all functions of the form

$$
\bar{P} R_{z} \bar{P} y=\sum_{i=0}^{\infty} \bar{P} R_{\zeta}^{\prime i+1} \bar{P}(z-\zeta)^{i} y, \quad y \in \bar{\chi}_{0}^{\prime},
$$

which are completely characterized by their first terms

$$
\bar{P} R_{\zeta}^{\prime} \bar{P} y,
$$

since $\bar{\chi}_{1}^{\prime}$ contains all elements $y$ of $\bar{\chi}^{\prime}$ for which $\bar{P}^{\prime} R_{\dot{q}}^{\prime} \bar{P}^{\prime} y$ is zero.

III. The set of all functions of the form

$$
\bar{P} R_{z} \bar{P} y=\sum_{i=1}^{\infty} \bar{P}\left(R_{\zeta}^{\prime}\right)^{i+1} \bar{P}(z-\zeta)^{i} y, \quad y \in \bar{\chi}_{1}^{\prime},
$$

which are also characterized by their first terms 


$$
(z-\zeta) \bar{P} R_{\zeta}^{\prime 2} \bar{P} y,
$$

since, as we shall show right now, $\bar{P} R_{\zeta}^{\prime 2} \bar{P} y=0, y \in \bar{\chi}_{1}^{\prime}$ if and only if $y=0$.

We are going to show now that the expression

(13) $\bar{P} P_{\zeta} \bar{P} y_{1}+\bar{P} R_{\zeta}^{\prime} \bar{P} y_{2}+\bar{P} R_{\zeta}^{\prime 2} \bar{P} y_{3}$, with $y_{1} \in \bar{\chi}^{\prime \prime}, y_{2} \in \bar{\chi}_{0}^{\prime}, y_{3} \in \bar{\chi}_{1}^{\prime}$,

vanishes if and only if $y_{1}=y_{2}=y_{3}=0$. For $\operatorname{Im} \zeta \neq 0$, the subspaces $H_{\zeta}$ and $\chi_{\zeta}$ are empty, since $Q$ and $P Q P$ are self-adjoint operators. The subspaces $\bar{\chi}^{\prime \prime}$ and $\bar{\chi}_{1}^{\prime}$ of $\bar{\chi}$ are then empty ${ }^{2}$ and $\bar{\chi}=\bar{\chi}_{0}$ so that our proposition becomes obvious for $\operatorname{Im} \zeta \neq 0$. Let us assume now that $\zeta$ is real. Since $y_{2}$ and $y_{3}$ are elements of $\bar{\chi}^{\prime}=\bar{\chi} \cap \bar{H}_{\zeta}$ and since $\bar{P} P_{\zeta} \bar{P} y_{1}$ $\in \bar{\chi}^{\prime \prime}$, the expression (13) vanishes only if

$$
\bar{P}^{\prime} R_{\zeta}^{\prime} \bar{P}^{\prime} y_{2}+\bar{P}^{\prime} R_{\zeta}^{\prime 2} \bar{P}^{\prime} y_{3}=0 .
$$

From this equality we can deduce

$$
\left(y_{3}, \bar{P}^{\prime} R_{\zeta}^{\prime} \bar{P}^{\prime} y_{2}\right)+\left(y_{3}, \bar{P}^{\prime} R_{\zeta}^{\prime 2} \bar{P}^{\prime} y_{3}\right)=0 .
$$

But since

$$
\left(y_{3}, \bar{P}^{\prime} R_{\zeta}^{\prime} \bar{P}^{\prime} y_{2}\right)=\left(\bar{P}^{\prime} R_{\zeta}^{\prime} \bar{P}^{\prime} y_{3}, y_{2}\right)=\left(0, y_{2}\right)=0,
$$

and

$$
\left(y_{3}, \bar{P}^{\prime} R_{\zeta}^{\prime 2} \bar{P}^{\prime} y_{3}\right)=\left(R_{\zeta}^{\prime} y_{3}, R_{\zeta}^{\prime} y_{3}\right),
$$

we must have $R_{\zeta}^{\prime} y_{3}=0$ which would imply $y_{3}=\bar{P}_{\zeta} y_{3}=\left(Q-I_{\zeta}\right) R_{\zeta}^{\prime} y_{3}=0$. On the other hand, $\bar{P}^{\prime} R_{\zeta}^{\prime} \bar{P}^{\prime} y_{2}\left(y_{2} \in \bar{\chi}_{0}^{\prime}\right)$ vanishes if and only if $y_{2}=0$. The vanishing of (13) would therefore imply $y_{2}=y_{3}=0$, and $\bar{P} P_{\zeta} \bar{P} y_{1}$ $=0$ which implies $y_{1}=0$.

We can now formulate the result of our analysis as follows:

Theorem. The subspaces $H_{\zeta} \ominus\left(H_{\zeta} \cap \chi\right)$ and $\chi_{\zeta} \theta\left(H_{\zeta} \cap \chi\right)$ are respectively equal to

$$
P_{\zeta} \bar{\chi}^{\prime \prime} \quad \text { and }\left(R_{\zeta}^{\prime}-T \bar{P}^{\prime \prime} R_{\zeta}^{\prime}\right) \bar{\chi}_{1}^{\prime}
$$

with $\bar{\chi}^{\prime \prime} \oplus \bar{\chi}_{0}^{\prime} \oplus \bar{\chi}_{1}^{\prime}=\bar{\chi}$, where $\bar{\chi}^{\prime}=\bar{\chi}_{0}^{\prime} \oplus \bar{\chi}_{1}^{\prime}$ is the subspace of $\bar{\chi}$ for which $\bar{P} R_{z} \bar{P}$ is regular at the point $z=\zeta$ and $\bar{\chi}_{1}^{\prime}$ the subspace for which $\bar{P} R_{z} \bar{P}$ has a zero at $z=\zeta$. The correspondences defined by the operators $P_{\zeta}, R_{\zeta}^{\prime}$ $-T \bar{P}^{\prime \prime} R_{\zeta}^{\prime}$ between

$$
\begin{array}{lll}
\bar{\chi}^{\prime \prime} \text { and } P_{\zeta} \bar{\chi}^{\prime \prime}, \\
\bar{\chi}_{1}^{\prime} \text { and }\left(R_{\zeta}^{\prime}-T \bar{P}^{\prime \prime} R_{\zeta}^{\prime}\right) \bar{\chi}_{1}^{\prime},
\end{array}
$$

respectively, are one-to-one correspondences. The subspaces of the co-

${ }^{2}$ Since $\bar{\chi}^{\prime \prime}$ and $\bar{\chi}_{1}^{\prime}$, are respectively isomorphic to $H_{\zeta} \ominus\left(H_{\zeta} \cap_{\chi}\right)$ and $\chi \zeta \ominus\left(H_{\zeta} \cap_{\chi}\right)$. 
efficients of the first terms of the functions

$$
\begin{aligned}
& y_{1}(z)=\bar{P} R_{z} \bar{P} y_{1}, \\
& y_{1} \in \bar{\chi}^{\prime \prime}, \\
& y_{2}(z)=\bar{P} R_{z} \bar{P} y_{2}, \\
& y_{2} \in \bar{\chi}_{0}^{\prime} \text {, } \\
& y_{3}(z)=\bar{P} R_{z} \bar{P} y_{3}, \\
& y_{3} \in \bar{\chi}_{1}^{\prime} \text {, }
\end{aligned}
$$

are the three linearly independent subspaces

$$
\bar{P} P_{\zeta} \bar{P} \bar{\chi}^{\prime \prime}, \quad \bar{P} R_{\zeta}^{\prime} \bar{P} \bar{\chi}_{0}^{\prime}, \quad \bar{P} R_{\zeta}^{\prime 2} \bar{P} \bar{\chi}_{1}^{\prime}
$$

of $\bar{\chi}$ which are respectively isomorphic to $\bar{\chi}^{\prime \prime}, \bar{\chi}_{0}^{\prime}, \bar{\chi}_{1}^{\prime}$.

This statement includes the main known property of Weinstein's determinant. In fact let us consider the special case where the subspace $\bar{\chi}$ is finite-dimensional. Then

$$
\begin{gathered}
H_{\zeta} \theta\left(H_{\zeta} \cap \chi\right)=P_{\zeta} \bar{\chi}^{\prime \prime}, \quad \chi_{\zeta} \theta\left(H_{\zeta} \cap \chi\right)=\left(R_{\zeta}^{\prime}-T \bar{P}^{\prime \prime} R_{\zeta}^{\prime}\right) \bar{\chi}_{1}^{\prime}, \\
\bar{\chi}^{\prime \prime}, \quad \bar{\chi}_{0}^{\prime}, \quad \bar{\chi}_{1}^{\prime}, \\
\bar{P} P_{\zeta} \bar{P} \bar{\chi}^{\prime \prime}, \quad \bar{P} R_{\zeta}^{\prime} \bar{P} \bar{\chi}_{0}^{\prime}, \quad \bar{P} R_{\zeta}^{\prime 2} \bar{P} \bar{\chi}_{1}^{\prime}
\end{gathered}
$$

are finite too and our theorem states that

$$
\begin{gathered}
\operatorname{dim}\left(H_{\zeta} \ominus\left(H_{\zeta} \cap \chi\right)\right)=\operatorname{dim} \bar{\chi}^{\prime \prime}, \\
\operatorname{dim}\left(\chi_{\zeta} \theta\left(H_{\zeta} \cap \chi\right)\right)=\operatorname{dim} \bar{\chi}_{1}^{\prime}, \\
\bar{\chi}=\bar{\chi}^{\prime \prime} \oplus \bar{\chi}_{0}^{\prime} \oplus \bar{\chi}_{1}^{\prime}=\bar{P} P_{\zeta} \bar{P} \bar{\chi}^{\prime \prime}+\bar{P} P_{\zeta}^{\prime} \bar{P} \bar{\chi}_{0}^{\prime}+\bar{P} R_{\zeta}^{\prime 2} \bar{P} \bar{\chi}_{1}^{\prime}
\end{gathered}
$$

with

$$
\begin{aligned}
& \nu^{\prime \prime}=\operatorname{dim} \bar{\chi}^{\prime \prime}=\operatorname{dim} \bar{P} P_{\zeta} \bar{P} \bar{\chi}^{\prime \prime}, \\
& \nu_{0}^{\prime}=\operatorname{dim} \bar{\chi}_{0}^{\prime}=\operatorname{dim} \bar{P} R_{\zeta}^{\prime} \bar{P} \bar{\chi}_{0}^{\prime}, \\
& \nu_{1}^{\prime}=\operatorname{dim} \bar{\chi}_{1}^{\prime}=\operatorname{dim} \bar{P} R_{\zeta}^{\prime 2} \bar{P} \bar{\chi}_{1}^{\prime} .
\end{aligned}
$$

Let $e_{1}^{\prime \prime}, e_{2}^{\prime \prime}, \cdots, e_{\nu^{\prime \prime}}^{\prime \prime} ; e_{1}^{\prime}(0), e_{2}^{\prime}(0), \cdots, e_{\nu_{0}}^{\prime}(0) ; e_{1}^{\prime}(1), e_{2}^{\prime}(1), \cdots$, $e_{\nu_{1}}^{\prime}(1)$ be a system of orthonormal bases of $\bar{\chi}^{\prime \prime}, \bar{\chi}_{0}^{\prime}, \bar{\chi}_{1}^{\prime}$ respectively. The Weinstein determinant $\left|\bar{P} R_{z} \bar{P}\right|$ will have the following expression:

$$
\begin{aligned}
\left|\bar{P} R_{z} \bar{P}\right| & \equiv\left|\begin{array}{l}
\bar{P} R_{z} \bar{P} e_{i}^{\prime \prime} \\
\bar{P} R_{z} \bar{P} e_{i}^{\prime}(0) \\
\bar{P} R_{z} \bar{P} e_{i}^{\prime}(1)
\end{array}\right| \\
& =(z-\zeta)^{\prime n^{\prime}-v^{\prime \prime}}\left\{\left|\begin{array}{c}
-\bar{P} P_{\zeta} \bar{P} e_{i}^{\prime \prime} \\
\bar{P} R_{\zeta}^{\prime} \bar{P} e_{i}^{\prime}(0) \\
\bar{P} R_{\zeta}^{\prime 2} \bar{P} e_{i}^{\prime}(1)
\end{array}\right|+(z-\zeta) A_{1}+\cdots\right\}
\end{aligned}
$$


where

$$
\left|\begin{array}{c}
-\bar{P} P_{\zeta} \bar{P} e_{i}^{\prime} \\
\bar{P} R_{\zeta}^{\prime} \bar{P} e_{i}^{\prime}(0) \\
\bar{P} R_{\zeta}^{\prime 2} \bar{P} e_{i}^{\prime}(1)
\end{array}\right| \neq 0,
$$

since the vectors $\bar{P} P_{\zeta} \bar{P} e_{1}^{\prime \prime}, \cdots, \quad \bar{P} P_{\zeta} \bar{P} e_{\nu^{\prime \prime}}^{\prime \prime} ; \quad \bar{P} R_{\zeta}^{\prime} \bar{P} e_{1}^{\prime}(0), \cdots$, $\bar{P} R_{\zeta}^{\prime} \bar{P} e_{\nu_{0}^{\prime}}^{\prime}(0) ; \bar{P} R_{\zeta}^{\prime 2} \bar{P} e_{1}^{\prime}(1), \cdots, \bar{P} R_{\zeta}^{\prime}{ }^{2} \bar{P} e_{\nu_{1}^{\prime}}^{\prime}(1)$ are linearly independent. The order $\nu_{\zeta}\left(\left|\bar{P} R_{z} \bar{P}\right|\right)$ of the function $\left|\bar{P} R_{z} \bar{P}\right|$ at the point $z=\zeta$ is therefore

$$
\begin{aligned}
\nu_{\zeta}\left(\left|\bar{P} R_{z} \bar{P}\right|\right) & =\operatorname{dim} \bar{\chi}_{1}^{\prime}-\operatorname{dim} \bar{\chi}^{\prime \prime} \\
& =\operatorname{dim}\left(\chi_{\zeta} \ominus\left(\chi \cap H_{\zeta}\right)\right)-\operatorname{dim}\left(H_{\zeta} \ominus\left(\chi \cap H_{\zeta}\right)\right) .
\end{aligned}
$$

This is the main property of Weinstein's determinant as stated by Aronszajn.

2. Aronszajn's problem. Let us consider now the following problem which is, in a certain way, the inverse of the preceding:

We assume that we know the resolvent

$$
\rho_{z}=-\frac{\pi_{\zeta}}{z-\zeta}+\sum_{i=0}^{\infty} \rho_{\zeta}^{\prime i+1}(z-\zeta)^{i}
$$

of $(P Q P-I z)$ in the neighborhood of the point $z=\zeta$ in the subspace $\chi$ and we want to determine the subspace $H_{\zeta}$ of all solutions $x$ of

$$
(Q-I \zeta) x=0
$$

in $H$. Here $\pi_{\xi}$ represents the projector associated with $\chi_{\zeta}$, that is, $\chi_{\zeta}=\pi_{\zeta} H$. We shall designate by $\bar{\pi}_{\zeta}$ the projector associated with $\bar{\chi}_{5}=\chi \ominus \chi_{5}$ and we shall have

$$
\begin{aligned}
\rho_{z}(P Q P-P z) & =(P Q P-P z) \rho_{z}=P, \\
\rho_{\zeta}^{\prime}(P Q P-P \zeta) & =(P Q P-P \zeta) \rho_{\zeta}^{\prime}=\bar{\pi}_{\zeta} .
\end{aligned}
$$

We consider again the identity (3). The first two terms of the left side of this identity are vectors of the subspace $\chi$ while the last two terms are vectors of the subspace $\bar{\chi} \cdot(Q-I \zeta) x$ will therefore vanish if and only if

$$
\begin{aligned}
& P(Q-I \zeta) P x+P(Q-I \zeta) \bar{P} x=0 \\
& \bar{P}(Q-I \zeta) P x+\bar{P}(Q-I \zeta) \bar{P} x=0 .
\end{aligned}
$$

Thus the solutions of (14) are furnished by

$$
x=P x+\bar{P} x
$$


where $\{P x, \bar{P} x\}$ is a solution of the system of two linear equations (15), (16) with the unknowns $P x$ and $\bar{P} x$. Let us assume that $\zeta$ is not an eigenvalue of $P Q P$. The projector $\pi_{\zeta}$ is then zero and $P(Q-I \zeta) P$ has a known inverse

$$
\rho_{\zeta}=\rho_{\zeta}^{\prime}
$$

in $\chi$. We can solve in this case the equation (15) with respect to $P x$ and substitute in (16). In this way we get

$$
\begin{gathered}
P x=-\rho_{\zeta} P(Q-I \zeta) \bar{P} x \\
-\bar{P}(Q-I \zeta) \rho_{\zeta} P(Q-I \zeta) \bar{P} x+\bar{P}(Q-I \zeta) \bar{P} x=0
\end{gathered}
$$

The function

$$
\begin{aligned}
& -\bar{P}(Q-I z) \rho_{z} P(Q-I z) \bar{P} x+\bar{P}(Q-I z) \bar{P} x \\
& =\bar{P}\left(I-(Q-I z) \rho_{z} P\right)(Q-I z) \bar{P} x=\bar{P}(Q-I z)\left(I-\rho_{z} P(Q-I z)\right) \bar{P} x
\end{aligned}
$$

has therefore a zero at $z=\zeta$ for $\bar{P} x \in \bar{P} H_{\zeta}=\bar{\chi}^{\prime \prime}$. But every element of $\bar{\chi}^{\prime \prime}$ is the coefficient of the principal part of a function of the form $\bar{P} R_{z} \bar{P} x$ which has a simple pole of first order at the point $z=\zeta$ so that we can expect that the product

$$
\bar{P}\left(I-(Q-I z) \rho_{z} P\right)(Q-I z) \bar{P} \cdot \bar{P} R_{z} \bar{P}
$$

has no pole at all at the point $z=\zeta$. In fact this product can be computed as follows:

$$
\begin{aligned}
\bar{P}(I- & \left.(Q-I z) \rho_{z} P\right)(Q-I z) \bar{P} R_{z} \bar{P} \\
& =\bar{P}\left(I-(Q-I z) \rho_{z} P\right)(Q-I z)\left(I-P R_{z}\right) \bar{P} \\
& =\bar{P}\left(I-(Q-I z) \rho_{z} P\right)(Q-I z)\left(R_{z}-P R_{z}\right) \bar{P} \\
& =\bar{P}\left(I-(Q-I z) \rho_{z} P\right)\left(I-(Q-I z) P R_{z}\right) \bar{P} \\
& =\bar{P}\left(I-(Q-I z) P R_{z}-(Q-I z) \rho_{z} P+(Q-I z) P R_{z}\right) \bar{P}=\bar{P} .
\end{aligned}
$$

One can verify in the same way that

$$
\bar{P} R_{z} \bar{P} \cdot \bar{P}(Q-I z)\left(I-\rho_{z} P(Q-I z)\right) \bar{P}=\bar{P} .
$$

The operators $\bar{P} R_{z} \bar{P}$ and

$-\bar{P} \cdot(Q-I z) \rho_{z} P(Q-I z) \bar{P}+\bar{P}(Q-I z) \bar{P}=\bar{P}\left(Q-I z-Q \rho_{z} P Q\right) \bar{P}$

are therefore the inverse of each other in $\bar{\chi}$.

This proposition contains the known Aronszajn result that in the case where $\bar{\chi}$ is finite, the determinant $\left|\bar{P}\left(Q-I z+Q \rho_{x} Q\right) \bar{P}\right|$ is the inverse of the Weinstein determinant $\left|\bar{P} R_{z} \bar{P}\right|$. 


\section{REFERENCES}

1. N. Aronszajn, Rayleigh-Ritz and A. Weinstein methods for approximation of eigenvalues, Proc. Nat. Acad. Sci. U.S.A. vol. 34 (1948) pp. 474-480 and 594-601.

2. A. Weinstein, New methods for the approximation of eigenvalues, Lectures at the University of Maryland, 1950-1951 (in preparation).

UNIVERSITY OF MARYLAND AND

UNIVERSITY OF ISTANBUL

\section{ON SOME FUNCTIONS HOLOMORPHIC IN AN INFINITE REGION}

\section{YU CHIA-YUNG ${ }^{1}$}

S. Mandelbrojt indicated the following proposition: If a function is holomorphic and bounded in a half-strip of the $z$-plane containing the half-axis $o x$ as a part of its central line and if this function and a certain infinite sequence of its derivatives vanish at the origin, then it is identically zero. The proof of this proposition is based upon a result of Mandelbrojt [1, p. 372]. ${ }^{2}$ In the present paper, we consider a function $F(z)$ holomorphic in a region $\Delta$ of the $z$-plane defined by $x \geqq d,|y| \leqq g(x)$, where $-\infty<d<0$ and where $g(x)$ is a certain positive continuous function tending to zero with $1 / x$. In this case if, in $\Delta, F(z)$ tends to zero rapidly enough and uniformly with respect to $y$ as $x$ tends to infinity, and if $F(z)$ and a certain infinite sequence of its derivatives vanish at the origin, then $F(z)$ is identically zero. In order to establish our proposition, we prove at first a lemma by means of the following theorem of $G$. Valiron $[3$, p. $62, \S 32]$ :

TheORem V. Let $Y(X)$ be a real function having a first derivative for $X \geqq X_{0}$ such that

$$
\lim _{x=\infty} \frac{X Y^{\prime}(X)}{\psi(X)}=1 ; \quad \psi(X) \geqq 1, \quad X \geqq X_{0} ; \quad \lim _{x=\infty} \frac{X \psi^{\prime}(X)}{[\psi(X)]^{2}}=0 .
$$

Let $\Phi(X)$ be an entire function and let $M(r)=\max _{|z|-r}|\Phi(z)|$. Then $a$ necessary and sufficient condition that

Presented to the Society, September 7, 1951; received by the editors May 1, 1951.

${ }^{1}$ The author wishes to express to Professors S. Mandelbrojt and G. Valiron his respectful gratitude for their kind and precious suggestions and criticisms.

2 Numbers in brackets refer to the bibliography at the end of this paper. 